\title{
Using Fluorine-ion Implanted a-Si Layer to Reduce Ni Contamination and Passivate the Defects in NILC poly-Si
}

\author{
Chien-Chih Chen, YewChung Sermon Wu
}

\begin{abstract}
Department of Materials Science and Engineering, National Chiao Tung University, Hsinchu 30010, Taiwan, R.O.C
\end{abstract}

\begin{abstract}
The grain boundaries which included dangling bonds in Ni-metalinduced lateral crystallization (NILC) poly-Si TFT would trap Ni and $\mathrm{NiSi}_{2}$ precipitates. This phenomenon resulted in threshold voltage shifting and lower field-effect mobility. To resolve this issue, the a-Si layer with fluorine-ion implanted was used as gettering layer to reduce $\mathrm{Ni}$ contamination and passivate the dangling bonds in the active layer. It was found that the F-G layer could not only reduce the Ni contamination but also passivate the defects in NILC poly-Si.
\end{abstract}

\section{Introduction}

Polycrystalline silicon thin-film transistors (poly-Si TFTs) have been widely used in active matrix organic liquid crystal displays (AMOLCDs) because their higher carrier mobility and lower threshold voltage than conventional amorphous thin-film transistors (a-Si TFTs) (1). Ni-metal-induced lateral crystallization (NILC) is one of these effective methods that can reduce the crystallization temperature to fabricate poly-Si TFTs on inexpensive glass substrates (2-3). However, the poly-Si grain boundaries which included dangling bonds would trap $\mathrm{Ni}$ and $\mathrm{NiSi}_{2}$ precipitates. This phenomenon resulted in threshold voltage shifting and lower field-effect mobility (4-5). In order to reduce the $\mathrm{Ni}$ contamination and passivate the dangling bonds in the active layer, the a-Si layer with fluorine-ion implanted was used as gettering layer (F-G layer) to resolve the issue. In the gettering process, $\mathrm{Ni}$ precipitates would diffuse to the fluorine-ion implanted a-Si layer because of the concentration gradation difference (6). Concurrently, fluorine-ion also diffused into the active layer and passivated the dangling bonds which associated with trap states density (7).

\section{Experiment}

First, a 100 -nm-thick a-Si film was fabricated on a 500 -nm-thick $\mathrm{SiO}_{2}$ buffer layer. Then the photoresist was patterned to form desired $\mathrm{Ni}$ lines, and a 5-nm-thick Ni film was deposited on the a-Si, subsequently annealed at $530^{\circ} \mathrm{C}$ for 48 hours to form the NILC poly-Si film, as shown in Fig.1 (a). The unreacted Ni metal was removed by chemical etching. Next a $\sim 5$-nm-thick chemical oxide was formed on the NILC poly-Si film by a mixed solution of $\mathrm{H}_{2} \mathrm{SO}_{4}$ and $\mathrm{H}_{2} \mathrm{O}_{2}$ for 10 min, and a 100 -nm-thick a-Si film was subsequently deposited on the chemical oxide layer. To provide passivation effect for the NILC poly-Si layer, the fluorine-ion was implanted into the a-Si film (called F-G layer), as shown in Fig.1 (b). The accelerating energy was $42 \mathrm{keV}$ and the dosage of fluorine-ion was $5 \times 10^{14} \mathrm{~cm}^{-2}$. The F-G layer served as a gettering layer, while the middle chemical oxide film was used as an etching stop layer. Fig. 1 (c) showed when the gettering process carried out at $550^{\circ} \mathrm{C}$ for 12 hours, Ni precipitates would diffuse to the F-G layer 
because of the concentration gradation difference. The Ni precipitates which diffuse into F-G layer would induce the a-Si of F-G layer crystallization, called gettering induce NILC (GINILC). Concurrently, fluorine-ion also diffused into the NILC poly-Si layer and. Finally, the F-G gettering layer and the chemical oxide layer were both removed, lifted Ni reducd and defects passivated NILC-poly-Si film, as shown in Fig. 1 (d).

\section{Results and Discussions}

Fig. 2 showed the OM images of F-G layer transformation on the NILC-poly-Si film at gettering process. The Ni pad vestige and the NILC region of NILC poly-Si film could be seen in Fig. 2(a) when not annealed yet. After annealing for 6 hours, F-G layer appeared GINILC region which corresponded to the $\mathrm{Ni}$ pad round and NILC/NILC boundary in the NILC-poly-Si film, as shown in Fig. 2 (b). This phenomenon indicated the Ni precipitates in the NILC-poly-Si film indeed diffused from NILC-poly-Si layer to F-G layer because of the concentration gradation difference and caused GINILC in the F$\mathrm{G}$ film (6). On the other hand, the location of GINILC start in the F-G layer also implied the Ni contamination in the NILC-poly-Si film primary happened in the Ni pad round and NILC/NILC boundary. For NILC poly-Si TFT devices, if the channel included the NILC/NILC boundary, the excess Ni contamination would create the path of leakage current and rise off-current substantially. Fig. 2(c) showed the F-G layer annealing for 12 hours, the GINILC region in the F-G layer almost covered the NILC region in NILCpoly-Si layer. This revealed the Ni precipitates in NILC-poly-Si layer were quite a lot and enough to proceed with NILC.

Fig. 3 showed the secondary-ion mass spectroscopy (SIMS) analysis of NILC-poly-Si layer of the fluorine, silicon, and oxygen atoms. It was found that the fluorine-ion substantial diffused into NILC-poly-Si layer and accumulated at the interface. The fluorine-ion in NILC-poly-Si layer would passivate the defects such as dangling bonds which existed in grain boundaries, resulted in NILC poly-Si TFT devices electrical characteristic enhancement (7).

\section{Conclusions}

The a-Si layer with fluorine-ion implanted was used as gettering layer to reduce $\mathrm{Ni}$ contamination and passivate the dangling bonds in the active layer. It was found that during the gettering process, not only Ni diffused from NILC poly-Si layer to F-G layer which resulted in GINILC, but also fluorine-ion could diffuse into NILC poly-Si layer and passivate the defects in poly-Si grain boundaries. 
(a)

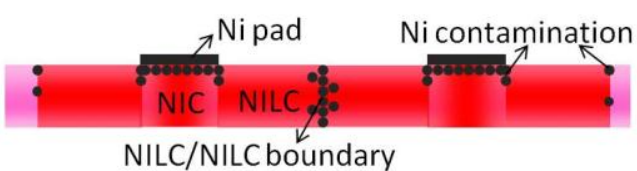

(c)

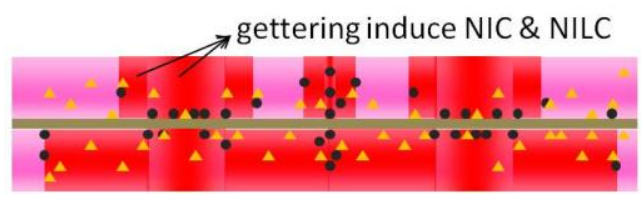

(b)

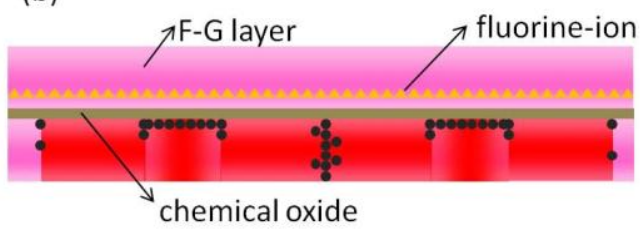

(d)

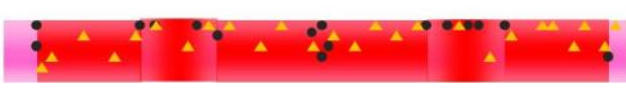

Fig. 1 The schematic illustration of the NILC \& gettering process: (a) the NILC process, (b) fabrication of F-G layer, (c) the gettering process, and (d) the Ni reduced and defects passivated NILC poly-Si film.
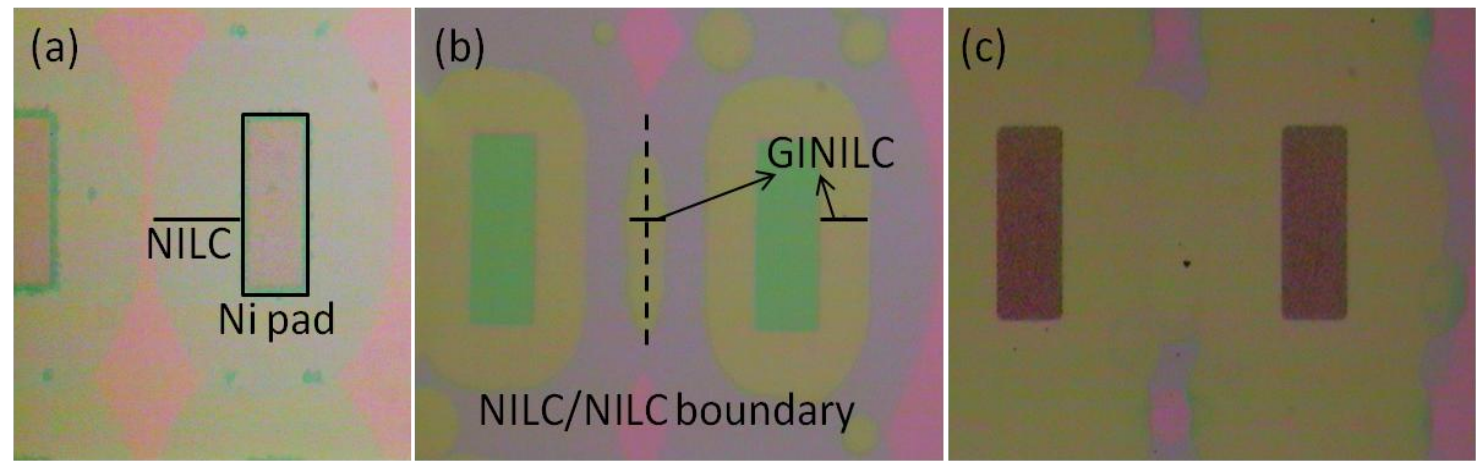

Fig. 2 The OM images of F-G layer transformation on the NILC-poly-Si film at gettering process: (a) before annealing, (b) annealing 6 hours, (c) annealing 12 hours.

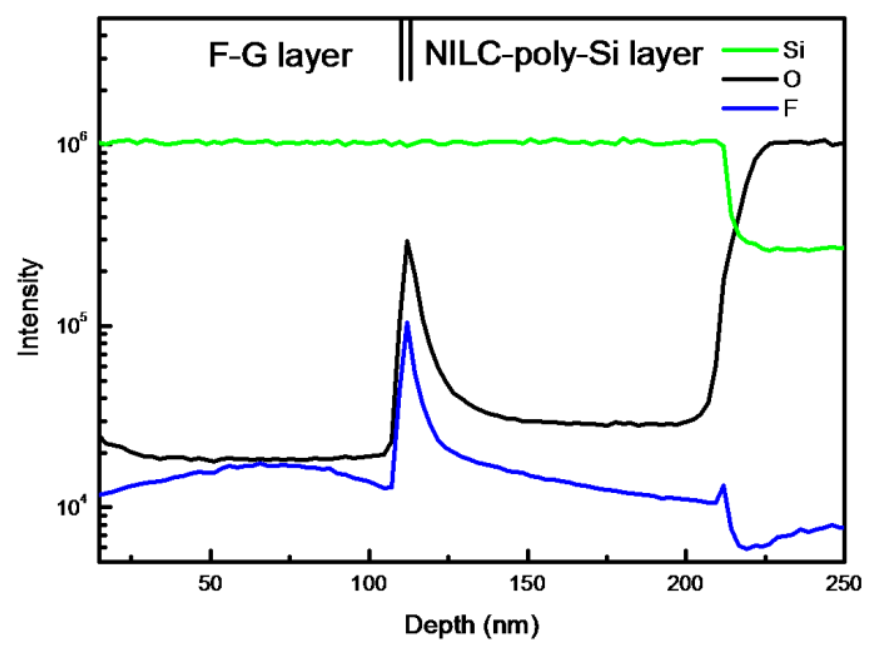

Fig 3. The SIMS depth profile of NILC poly-Si film of the fluorine, silicon, and oxygen atoms 


\section{Acknowledgments}

This project was funded by Sino American Silicon Products Incorporation and the National Science Council of the Republic of China under Grant No. 98-2221-E-009-041MY3. Technical supports from the National Nano Device Laboratory, Center for Nano Science and Technology and the Nano Facility Center of the National Chiao Tung University are also acknowledged.

\section{References}

1. M. Stewart, R. S. Howell, L. Pires, and M. K. Hatalis, IEEE Trans. Electron Devices, 48, 845 (2001)

2. S. W. Lee and S. K. Joo, IEEE Electron Device Lett., 17, 160 (1996).

3. Z. Meng, M. Wang, and M. Wong, IEEE Trans. Electron Devices, 47, 404 (2000).

4. P. J. van der Zaag, M. A. Verheijen, S. Y. Yoon, and N. D. Young, Appl. Phys. Lett., 81, 3404 (2002).

5. G. A. Bhat, Z. Jin, H. S. Kwok, and M. Wong, IEEE Electron Device Lett., 20, 97 (1999).

6. B. M. Wang and Y. C. Sermon Wu, Electrochem. Solid-State Lett., 12, J14 (2009).

7. C. P. Chang and Y. C. Sermon Wu, IEEE Electron Device Lett., 28, 990 (2007). 